\title{
A Triangular Spectral Method for the Stokes Equations
}

\author{
Lizhen Chen ${ }^{1}$, Jie Shen ${ }^{1,2}$ and Chuanju $\mathrm{Xu}^{1, *}$ \\ ${ }^{1}$ School of Mathematical Sciences, Xiamen University, 361005 Xiamen, China. \\ ${ }^{2}$ Department of Mathematics, Purdue University, West Lafayette, IN, 47907, USA. \\ Received 30 September 2010; Accepted (in revised version) 16 November 2010 \\ Available online 6 April 2011
}

\begin{abstract}
A triangular spectral method for the Stokes equations is developed in this paper. The main contributions are two-fold: First of all, a spectral method using the rational approximation is constructed and analyzed for the Stokes equations in a triangular domain. The existence and uniqueness of the solution, together with an error estimate for the velocity, are proved. Secondly, a nodal basis is constructed for the efficient implementation of the method. These new basis functions enjoy the fully tensorial product property as in a tensor-produce domain. The new triangular spectral method makes it easy to treat more complex geometries in the classical spectral-element framework, allowing us to use arbitrary triangular and tetrahedral elements.
\end{abstract}

AMS subject classifications: 65N35, 65N22, 65F05, 35J05

Key words: Stokes equations, triangular spectral method, error analysis.

\section{Introduction}

The spectral-element method is a high-order variational method which combines the geometric flexibility of finite-elements with the high accuracy of spectral methods. It exhibits several favorable computational properties, such as the use of tensor products, naturally diagonal mass matrices, and suitability for parallel computation. However, in order to use fast tensor product summation, the standard spectral-element method is usually restricted to quadrilateral partitions, which are difficult to use for adaptive computation in complex geometries. One way to overcome this drawback is to allow triangular partitions, which are more flexible in handling complex geometries.

Existing spectral methods on triangle can be classified into three categories according to the class of functions used in the approximations: (i) approximations by polynomials in triangle through mapping (see, e.g., $[3,5,8,12,16]$ ); (ii) approximations by polynomials in triangle using special nodal points such as Fekete points (see, e.g., $[7,13,14,17]$ ); and (iii)

\footnotetext{
*Corresponding author. Email addresses: shen@math.purdue.edu (J. Shen), cjxu@xmu.edu.cn (C. Xu) 
approximations by non-polynomial functions in triangle (see, e.g., $[2,6,15]$ ). The triangular spectral methods based on polynomials were motivated by the classical result that any smooth function can be well approximated by polynomials. The orthogonal polynomials on triangle, often referred as Dubiner's basis in the spectral-element community, were constructed by Koornwinder [9] and by Dubiner [5], and implemented in the spectral-element package NekTar (cf. $[8,16]$ ). A drawback of this approach is that no suitable interpolation operator, i.e., no corresponding nodal basis, is available for the Dubiner's basis, which involves a wrapped product, instead of the tensor product, of the Jacobi polynomials used to define the basis functions. The lack of nodal basis makes it difficult to implement in a collocation framework. Recently, a triangular spectral method using rational polynomials was proposed and analyzed (cf. [15]). The rational basis functions are constructed through the Duffy mapping in the reference square, and are mutually orthogonal with respect to a suitable weight function. In particular, one can construct a nodal basis through the Duffy mapping which allows simple and efficient implementation as a usual nodal spectral-element method.

In this paper, we will consider a triangular spectral method based on the rational function approximation for the Stokes equations. First, we construct and analyze a new triangular spectral method for the Stokes equations. By using a compatible pair of spaces for the velocity and pressure, we establish the well-posedness of the discrete problem, and derive an error estimate for the velocity. Although no theoretical estimate is provided for the inf-sup constant, numerical tests are carried out to investigate its asymptotic behavior. Second, we introduce a nodal basis, the transformation of which is the tensor product of the standard Lagrangian polynomials defined on the rectangular Gauss-Lobatto points, with an exception corresponding to the "collapsed" side. The remarkable advantage of this basis is that it enjoys the fully tensorial-product property, and easy to implement in case of multi-elements. The availability of nodal triangular basis greatly enhances the geometrical flexibility of spectral-element method by allowing fully unstructured mesh.

The paper is organized as follows. In the next section, we present some preliminary results which will be used in the sequel. Then we construct the triangular spectral method for the Stokes equations, and study its well-posedness and error estimate in Section 3. In Section 4, we provide implementation details, with a particular attention to the construction of the nodal basis functions. Some numerical results and discussions are presented in Section 5 .

\section{Construction of the triangular spectral method}

Throughout this paper, we will use boldface letters to denote vectors and vector functions. Let $c$ stand for a generic positive constant independent of any functions and of any discretization parameters. We use the expression $A \lesssim B$ to mean that $A \leqslant c B$, and use the expression $A \cong B$ to mean that $A \lesssim B \lesssim A$. For a bounded domain $\Omega$ and a generic positive weight function $\omega$, we denote the inner product of $L_{\omega}^{2}(\Omega)$ by

$$
(u, v)_{\omega, \Omega}:=\int_{\Omega} u v \omega d \mathbf{x}
$$


and the associated norm by $\|\cdot\|_{\omega, \Omega}$. We use $H_{\omega}^{m}(\Omega)$ and $H_{0, \omega}^{m}(\Omega)$ to denote the usual weighted Sobolev spaces, whose norm and semi-norm are denoted by $\|u\|_{m, \omega, \Omega}$ and $|u|_{m, \omega, \Omega}$, respectively. We also denote

$$
L_{0, \omega}^{2}(\Omega)=\left\{\phi \in L_{\omega}^{2}(\Omega): \int_{\Omega} \phi \omega d \mathbf{x}=0\right\} .
$$

In cases with no confusion would arise, $\omega$ (if $\omega=1$ ) and/or $\Omega$ may be dropped from the notations. Let $\mathbb{N}$ be the set of all non-negative integers and $\Lambda=(-1,1)$. For any $N \in \mathbb{N}$, we denote by $\mathbb{P}_{N}(\Lambda)$ the set of all polynomials of degree $\leq N$ defined in $\Lambda$, and set $\mathbb{P}_{N}^{0}(\Lambda):=\left\{\phi \in \mathbb{P}_{N}(\Lambda): \phi( \pm 1)=0\right\}$.

\subsection{Preliminaries}

Let $\square:=(-1,1)^{2}$ and $\triangle$ be the triangular domain

$$
\triangle=\{(x, y): 0<x, y<1,0<x+y<1\} .
$$

We will use two coordinate systems: the Cartesian coordinate $(x, y)$-system for the triangle $\triangle$ and the $(\xi, \eta)$-system for the square $\square$. For ease of notation, we also denote $\mathbf{x}=(x, y)$ and $\xi=(\xi, \eta)$.

(a)

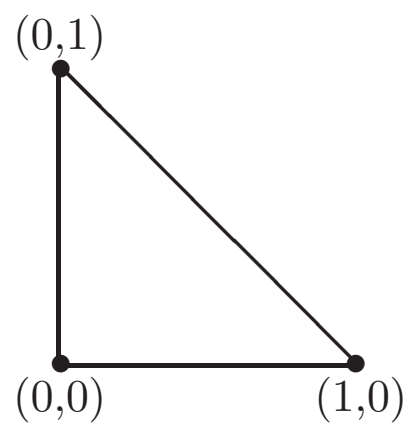

(b)

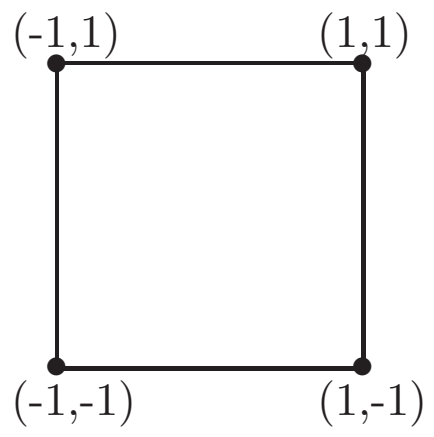

Figure 1: (a) Domain $\triangle$ with coordinate $(x, y)$. (b) Domain $\square$ with coordinate $(\xi, \eta)$.

An one-to-one transformation from $\square$ to $\triangle$ is given by the Duffy mapping $\mathbf{x}=\mathbf{F}(\xi)$ :

$$
\left\{\begin{array}{ll}
x & =\frac{1}{4}(1+\xi)(1-\eta), \\
y & =\frac{1+\eta}{2},
\end{array} \quad \forall(\xi, \eta) \in \square,\right.
$$

with its inverse $\xi=\mathbf{F}^{-1}(\mathbf{x})$ from $\triangle$ to $\square$ by

$$
\left\{\begin{array}{l}
\xi=\frac{2 x}{1-y}-1, \quad \forall(x, y) \in \triangle . \\
\eta=2 y-1,
\end{array}\right.
$$


$(\xi, \eta)$ is often referred to as collapsed coordinate system or the Duffy coordinates. Note that the transformation (2.1) maps the whole top side of $\square$ to the top vertex $(0,1)$ of $\triangle$, and the two vertical sides of $\square$ onto sides radiating out of this vertex.

We collect here some properties of the Duffy mapping, which will be used in the sequel.

$$
\frac{\partial \xi}{\partial x}=\frac{2}{1-y}=\frac{4}{1-\eta}, \frac{\partial \xi}{\partial y}=\frac{2 x}{(1-y)^{2}}=\frac{2(1+\xi)}{1-\eta}, \frac{\partial \eta}{\partial x}=0, \frac{\partial \eta}{\partial y}=2,
$$

and

$$
\frac{\partial x}{\partial \xi}=\frac{1-y}{2}=\frac{1-\eta}{4}, \frac{\partial x}{\partial \eta}=\frac{x}{2(1-y)}=-\frac{1+\xi}{4}, \frac{\partial y}{\partial \xi}=0, \frac{\partial y}{\partial \eta}=\frac{1}{2} .
$$

From the above, one easily finds the determinant of the Jacobian for (2.1):

$$
\operatorname{det}\left(\frac{\partial(x, y)}{\partial(\xi, \eta)}\right)=\frac{1-\eta}{8}=\frac{1-y}{4} .
$$

Throughout the paper, we shall associate a function $u$ in $\triangle$ with a function $\widetilde{u}$ in through

$$
\widetilde{u}(\xi, \eta)=u(x, y), x=\frac{1}{4}(1+\xi)(1-\eta), y=\frac{1+\eta}{2}, \quad \forall(\xi, \eta) \in \square .
$$

It is easy to see that

$$
\nabla_{\mathbf{x}} u=\left(\partial_{x} u, \partial_{y} u\right)^{T}=\left(\frac{4}{1-\eta} \partial_{\xi} \tilde{u}, \frac{2(1+\xi)}{1-\eta} \partial_{\xi} \tilde{u}+2 \partial_{\eta} \tilde{u}\right)^{T},
$$

and inversely,

$$
\nabla_{\xi} \widetilde{u}=\left(\partial_{\xi} \widetilde{u}, \partial_{\eta} \widetilde{u}\right)^{T}=\left(\frac{1-y}{2} \partial_{x} u, \frac{x}{2(1-y)} \partial_{x} u+\frac{1}{2} \partial_{y} u\right)^{T} .
$$

From (2.7), we have immediately

$$
\partial_{\xi} \widetilde{u}(\xi, 1)=0 \text { a.e. if } \partial_{x} u \text { is a measurable function. }
$$

In other words, if the approximation solution is chosen such that its partial derivative with respect to $x$ is bounded in $\triangle$, then its transformation in $\square$ by Duffy mapping must be constant at the top side. This fact should be kept in mind in constructing the basis functions for the approximation space.

The approximation space to be used in our method consists of the rational functions generated by polynomials in the reference square through the Duffy transform. More precisely, let $\widetilde{\mathscr{R}}_{m n}(\xi, \eta)$ be the polynomial in $\square$ defined by:

$$
\widetilde{\mathscr{R}}_{m n}(\xi, \eta)=J_{m}^{0,0}(\xi) J_{n}^{1,0}(\eta), \quad \forall(\xi, \eta) \in \square,
$$


where $J_{k}^{\alpha, \beta}(\zeta), \zeta \in \Lambda$, is the classical Jacobi polynomial of degree $k$. Then we define the rational function $\mathscr{R}_{m n}(x, y)$ in $\triangle$ by the Duffy transformation of $\widetilde{\mathscr{R}}_{m n}(\xi, \eta)$, i.e.,

$\mathscr{R}_{m n}(x, y)=\widetilde{R}_{m n}\left(\frac{2 x}{1-y}-1,2 y-1\right)=J_{m}^{0,0}\left(\frac{2 x}{1-y}-1\right) J_{n}^{1,0}(2 y-1), \quad \forall(x, y) \in \triangle$, and the approximation spaces and their transformations as follows:

$$
\begin{aligned}
& \mathbb{Q}_{N}(\triangle)=\operatorname{span}\left\{\mathscr{R}_{m n}(x, y), 0 \leq m, n \leq N,(x, y) \in \triangle\right\}, \\
& \widetilde{\mathbb{Q}}_{N}(\square)=\operatorname{span}\left\{\widetilde{R}_{m n}(\xi, \eta), 0 \leq m, n \leq N,(\xi, \eta) \in \square\right\}, \\
& \mathbb{Q}_{N}^{0}(\triangle)=\left\{v \in \mathbb{Q}_{N}(\triangle):\left.v\right|_{\partial \Delta}=0\right\}, \\
& \widetilde{\mathbb{Q}}_{N}^{0}(\square)=\left\{v \in \widetilde{\mathbb{Q}}_{N}(\square):\left.v\right|_{\partial \square}=0\right\} .
\end{aligned}
$$

By the orthogonality of the Jacobi polynomials, we have the following orthogonality:

$$
\begin{aligned}
& \int_{\triangle} \mathscr{R}_{m n}(x, y) \mathscr{R}_{m^{\prime} n^{\prime}}(x, y) d x d y \\
= & \frac{1}{8} \int_{-1}^{1} J_{m}^{0,0}(\xi) J_{m^{\prime}}^{0,0}(\xi) d \xi \int_{-1}^{1} J_{n}^{1,0}(\eta) J_{n^{\prime}}^{1,0}(\eta)(1-\eta) d \eta \\
= & \gamma_{m n} \delta_{m m^{\prime}} \delta_{n n^{\prime}}, \quad \text { with } \gamma_{m n}=\frac{1}{2(n+1)(2 m+1)} .
\end{aligned}
$$

Furthermore, any function $u \in L^{2}(\triangle)$ can be expressed as

$$
u(x, y)=\sum_{m=0}^{\infty} \sum_{n=0}^{\infty} \widehat{u}_{m n} \mathscr{R}_{m n}(x, y),
$$

with the coefficient $\widehat{u}_{m n}$ given by

$$
\widehat{u}_{m n}=\frac{1}{\gamma_{m n}} \int_{\triangle} u(x, y) \mathscr{R}_{m n}(x, y) d x d y .
$$

On the other hand, we have $\tilde{u} \in L_{\varpi}^{2}(\square)$ if and only if $u \in L^{2}(\triangle)$, where the weight function

$$
\varpi:=\frac{1-\eta}{8}
$$

is the Jacobian in (2.5), and

$$
\widetilde{u}(\xi, \eta)=\sum_{m=0}^{\infty} \sum_{n=0}^{\infty} \widehat{u}_{m n} \widetilde{\mathscr{R}}_{m n}(\xi, \eta),
$$

with $\widehat{u}_{m n}$ given in (2.11), or expressed in the alternative form:

$$
\widehat{u}_{m n}=\frac{1}{\gamma_{m n}} \int_{\square} \widetilde{u}(\xi, \eta) \widetilde{R}_{m n}(\xi, \eta) \varpi d \xi d \eta .
$$


It can be checked, by using (2.6) and (2.7), that the space associated to $H^{1}(\triangle)$ by the Duffy mapping is:

$$
\widetilde{H}_{\varpi}^{1}(\square):=\left\{\widetilde{u} \in L_{\varpi}^{2}(\square): \partial_{\xi} \widetilde{u} \in L_{\varpi^{-1}}^{2}(\square) \text { and } \partial_{\eta} \widetilde{u} \in L_{\varpi}^{2}(\square)\right\},
$$

endowed with the norm:

$$
\|\widetilde{u}\|_{\widetilde{H}_{\varpi}^{1}(\square)}=\left(\|\widetilde{u}\|_{L_{\varpi}^{2}(\square)}^{2}+\left\|\partial_{\xi} \widetilde{u}\right\|_{L_{\sigma^{-1}}^{2}(\square)}^{2}+\left\|\partial_{\eta} \widetilde{u}\right\|_{L_{\varpi}^{2}(\square)}^{2}\right)^{\frac{1}{2}} .
$$

Moreover, noticing that

$$
0 \leq \frac{x}{1-y}=\frac{1+\xi}{2} \leq 1, \quad \forall(x, y) \in \triangle \text { and }(\xi, \eta) \in \square,
$$

we have the following norm equivalence:

$$
\|u\|_{H^{1}(\triangle)} \cong\|\widetilde{u}\|_{\widetilde{H}_{\varpi}^{1}(\square)}
$$

\subsection{A triangular spectral method for the Stokes equations}

The Stokes equations in $\triangle$ are as follows: For a given forcing function $\mathbf{f} \in L^{2}(\triangle)^{2}$, find the velocity field $\mathbf{u}$ and the pressure $p$ such that

$$
\begin{cases}-\Delta \mathbf{u}+\nabla p=\mathbf{f}, & \text { in } \triangle, \\ \nabla \cdot \mathbf{u}=0, & \text { in } \triangle, \\ \mathbf{u}=\mathbf{0}, & \text { on } \partial \triangle .\end{cases}
$$

We shall first write (2.15) in an abstract weak formulation. Let $X=H_{0}^{1}(\triangle)$ with $\mathbf{X}=X^{2}$, and $M=L_{0}^{2}(\triangle)$. we introduce the bilinear form $a(\cdot, \cdot)$ over $\mathbf{X} \times \mathbf{X}$ by

$$
a(\mathbf{v}, \mathbf{w})=\int_{\triangle} \nabla \mathbf{v} \nabla \mathbf{w} d x d y, \quad \forall \mathbf{v}, \mathbf{w} \in \mathbf{X}
$$

and the bilinear form $b(\cdot, \cdot)$ over $\mathbf{X} \times M$ by,

$$
b(\mathbf{v}, q)=-\int_{\triangle} \nabla \cdot \mathbf{v} q d x d y, \quad \forall \mathbf{v} \in \mathbf{X}, q \in M .
$$

Then the weak formulation for the Stokes problem (2.15) reads: Find $\mathbf{u} \in \mathbf{X}$ and $p \in M$ such that

$$
\begin{cases}a(\mathbf{u}, \mathbf{v})+b(\mathbf{v}, p)=(\mathbf{f}, \mathbf{v}), & \forall \mathbf{v} \in \mathbf{X}, \\ b(\mathbf{u}, q)=0, & \forall q \in M .\end{cases}
$$


In order to construct our triangular spectral method, we let $\mathbf{X}_{N}$ and $M_{N}$ be the rational function spaces, defined by

$$
\mathbf{X}_{N}=X_{N}^{2}, \quad X_{N}=X \cap \mathbb{Q}_{N}(\triangle), M_{N}=M \cap \mathbb{Q}_{N-2}(\triangle),
$$

and $a_{N}(\cdot, \cdot)$ and $b_{N}(\cdot, \cdot)$ are two discrete forms, which we define below. Let $\xi_{p}, p=$ $0,1, \cdots, N$, be the Legendre-Gauss-Lobatto points associated to $L_{N}$, i.e., zeros of $(1-$ $\left.z^{2}\right) L_{N}^{\prime}(z) ; \omega_{p}, p=0,1, \cdots, N$, be the corresponding weights. Then, we have the wellknown Legendre Gauss-Lobatto quadrature:

$$
\int_{-1}^{1} \varphi(z) d z=\sum_{q=0}^{N} \varphi\left(\xi_{q}\right) \omega_{q}, \quad \forall \varphi \in \mathbb{P}_{2 N-1}(\Lambda)
$$

and the norm equivalence (cf. [4]):

$$
\sum_{q=0}^{N} \varphi^{2}\left(\xi_{q}\right) \omega_{q} \cong\|\varphi\|^{2}, \quad \forall \varphi \in \mathbb{P}_{N}(\Lambda)
$$

Thanks to the identity

$$
(\phi, \psi)=\int_{\triangle} \phi(x, y) \psi(x, y) d x d y=\int_{\square} \widetilde{\phi}(\xi, \eta) \widetilde{\psi}(\xi, \eta) \frac{1-\eta}{8} d \xi d \eta, \quad \forall \phi, \psi \in C(\triangle),
$$

where $\frac{1-\eta}{8}$ is nothing than the Jacobian given in (2.5), we can then define the discrete inner product $(\cdot, \cdot)_{N}$ on $\triangle$ :

$$
(\phi, \psi)_{N}=\sum_{p, q=0}^{N} \tilde{\phi}\left(\xi_{p}, \xi_{q}\right) \widetilde{\psi}\left(\xi_{p}, \xi_{q}\right) \frac{1-\xi_{q}}{8} \omega_{p} \omega_{q}, \quad \forall \phi, \psi \in C(\triangle),
$$

We now define the discrete bilinear forms:

$$
a_{N}\left(\mathbf{u}_{N}, \mathbf{v}_{N}\right)=\left(\nabla \mathbf{u}_{N}, \nabla \mathbf{v}_{N}\right)_{N}, \quad b_{N}\left(\mathbf{v}_{N}, q_{N}\right)=-\left(q_{N}, \nabla \cdot \mathbf{v}_{N}\right)_{N} .
$$

With the above definitions, our triangular spectral method for (2.16) is: Find $\mathbf{u}_{N} \in \mathbf{X}_{N}, p_{N} \in$ $M_{N}$, such that

$$
\begin{cases}a_{N}\left(\mathbf{u}_{N}, \mathbf{v}_{N}\right)+b_{N}\left(\mathbf{v}_{N}, p_{N}\right)=\left(\mathbf{f}, \mathbf{v}_{N}\right), & \forall \mathbf{v}_{N} \in \mathbf{X}_{N} \\ b_{N}\left(\mathbf{u}_{N}, q_{N}\right)=0, & \forall q_{N} \in M_{N} .\end{cases}
$$

Remark 2.1. It is well-known that, to avoid spurious pressure modes, the discrete velocity and pressure spaces for the Stokes equations are required to satisfy an inf-sup condition. In the above, similar to the usual $\mathbb{P}_{N} \times \mathbb{P}_{N-2}$ approach in a rectangular spectral method, we let the pressure approximation space $M_{N}$ in (2.17) to be two "degrees" less than the velocity approximation space $\mathbf{X}_{N}$. We will prove below that a discrete inf-sup condition is satisfied for this pair of spaces so the discrete problem (2.22) is well-posed. However, we are unable to derive a lower bound for the inf-sup constant and will resort to numerical experiments to study its behavior with respect to $N$. 


\section{Well-posedness and error estimation}

\subsection{Well-posedness of the discrete problem}

We shall prove the well-posedness of problem (2.22) by applying the classical saddlepoint theory (cf. [1]). The first task is to show that $a(\cdot, \cdot)$ is continuous and coercive over $\mathbf{X}_{N} \times \mathbf{X}_{N}$.

Lemma 3.1. There exist two positive constants $\alpha$ and $\gamma$ such that

$$
\left|a_{N}\left(\mathbf{u}_{N}, \mathbf{v}_{N}\right)\right| \leq \gamma\left\|\mathbf{u}_{N}\right\|_{1}\left\|\mathbf{v}_{N}\right\|_{1}, \quad \forall \mathbf{u}_{N}, \mathbf{v}_{N} \in \mathbf{X}_{N},
$$

and

$$
a_{N}\left(\mathbf{v}_{N}, \mathbf{v}_{N}\right) \geq \alpha\left\|\mathbf{v}_{N}\right\|_{1}^{2}, \quad \forall \mathbf{v}_{N} \in \mathbf{X}_{N} .
$$

Proof. By using (2.6) and (2.7), we have

$$
\begin{aligned}
& \int_{\triangle} \nabla_{\mathbf{x}} \mathbf{u}_{N}(x, y) \nabla_{\mathbf{x}} \mathbf{v}_{N}(x, y) d x d y \\
= & \int_{\square} \nabla_{\mathbf{x}} \mathbf{u}_{N} \circ \mathbf{F} \nabla_{\mathbf{x}} \mathbf{v}_{N} \circ \mathbf{F}|J| d \xi d \eta=\int_{\square} \widetilde{J}^{T} \nabla \xi \widetilde{\mathbf{u}}_{N} \widetilde{J}^{T} \nabla_{\xi} \widetilde{\mathbf{v}}_{N}|J| d \xi d \eta,
\end{aligned}
$$

where $\mathbf{F}$ is the Duffy mapping from $\triangle$ to $\square$ (see (2.1)), $J$ is its Jacobian:

$$
J=\left(\begin{array}{ll}
\frac{\partial x}{\partial \xi} & \frac{\partial x}{\partial \eta} \\
\frac{\partial y}{\partial \xi} & \frac{\partial y}{\partial \eta}
\end{array}\right)=\frac{1-\eta}{8}
$$

and $\widetilde{J}$ is the inverse of $J$ :

$$
\widetilde{J}=\left(\begin{array}{ll}
\frac{\partial \xi}{\partial x} & \frac{\partial \xi}{\partial y} \\
\frac{\partial \eta}{\partial x} & \frac{\partial \eta}{\partial y}
\end{array}\right)=\frac{1}{|J|}\left(\begin{array}{cc}
\frac{\partial x}{\partial \xi} & -\frac{\partial x}{\partial \eta} \\
-\frac{\partial y}{\partial \xi} & \frac{\partial y}{\partial \eta}
\end{array}\right) .
$$

A rearrangement of the above equality leads to

$$
\begin{aligned}
& \int_{\triangle} \nabla_{\mathbf{x}} \mathbf{u}_{N}(x, y) \nabla_{\mathbf{x}} \mathbf{v}_{N}(x, y) d x d y \\
= & \int_{\square}\left(G_{1} \frac{\partial \widetilde{\mathbf{u}}_{N}}{\partial \xi} \frac{\partial \widetilde{\mathbf{v}}_{N}}{\partial \xi}+G_{2} \frac{\partial \widetilde{\mathbf{u}}_{N}}{\partial \eta} \frac{\partial \widetilde{\mathbf{v}}_{N}}{\partial \eta}+G_{3}\left(\frac{\partial \widetilde{\mathbf{u}}_{N}}{\partial \xi} \frac{\partial \widetilde{\mathbf{v}}_{N}}{\partial \eta}+\frac{\partial \widetilde{\mathbf{u}}_{N}}{\partial \eta} \frac{\partial \widetilde{\mathbf{v}}_{N}}{\partial \xi}\right)\right) d \xi d \eta,
\end{aligned}
$$


where $G_{1}, G_{2}$, and $G_{3}$ are geometric factors, defined as

$$
\begin{aligned}
G_{1} & =\frac{1}{|J|}\left(\left(\frac{\partial x}{\partial \eta}\right)^{2}+\left(\frac{\partial y}{\partial \eta}\right)^{2}\right), \\
G_{2} & =\frac{1}{|J|}\left(\left(\frac{\partial x}{\partial \xi}\right)^{2}+\left(\frac{\partial y}{\partial \xi}\right)^{2}\right), \\
G_{3} & =-\frac{1}{|J|}\left(\frac{\partial x}{\partial \xi} \frac{\partial x}{\partial \eta}+\frac{\partial y}{\partial \xi} \frac{\partial y}{\partial \eta}\right) .
\end{aligned}
$$

Thus, by the definition (2.21), we have

$$
\begin{aligned}
a_{N}\left(\mathbf{u}_{N}, \mathbf{v}_{N}\right)=\sum_{p, q=}^{N} G_{1}\left(\xi_{p}, \xi_{q}\right) \frac{\partial \widetilde{\mathbf{u}}_{N}}{\partial \xi} \frac{\partial \widetilde{\mathbf{v}}_{N}}{\partial \xi}\left(\xi_{p}, \xi_{q}\right) \omega_{p} \omega_{q} \\
+\sum_{p, q=0}^{N} G_{2}\left(\xi_{p}, \xi_{q}\right) \frac{\partial \widetilde{\mathbf{u}}_{N}}{\partial \eta} \frac{\partial \widetilde{\mathbf{v}}_{N}}{\partial \eta}\left(\xi_{p}, \xi_{q}\right) \omega_{p} \omega_{q} \\
+\sum_{p, q=0}^{N} G_{3}\left(\xi_{p}, \xi_{q}\right)\left(\frac{\partial \widetilde{\mathbf{u}}_{N}}{\partial \xi} \frac{\partial \widetilde{\mathbf{v}}_{N}}{\partial \eta}+\frac{\partial \widetilde{\mathbf{u}}_{N}}{\partial \eta} \frac{\partial \widetilde{\mathbf{v}}_{N}}{\partial \xi}\right)\left(\xi_{p}, \xi_{q}\right) \omega_{p} \omega_{q}
\end{aligned}
$$

A direct calculation by using (2.4) and (2.5) gives

$$
\begin{aligned}
G_{1} & =\frac{8}{1-\eta} \frac{(1+\xi)^{2}+4}{16}=\frac{(1+\xi)^{2}+4}{2(1-\eta)}, \\
G_{2} & =\frac{8}{1-\eta} \frac{(1-\eta)^{2}}{16}=\frac{1-\eta}{2}, \\
G_{3} & =\frac{8}{1-\eta} \frac{(1+\xi)(1-\eta)}{16}=\frac{1+\xi}{2} .
\end{aligned}
$$

Thanks to the above and the fact that $\widetilde{\mathbf{u}}_{N}, \widetilde{\mathbf{v}}_{N} \in \widetilde{\mathbb{Q}}_{N}^{0}(\square)$, the three functions

$$
G_{1} \frac{\partial \widetilde{\mathbf{u}}_{N}}{\partial \xi} \frac{\partial \widetilde{\mathbf{v}}_{N}}{\partial \xi}, \quad G_{2} \frac{\partial \widetilde{\mathbf{u}}_{N}}{\partial \eta} \frac{\partial \widetilde{\mathbf{v}}_{N}}{\partial \eta}, \quad \text { and } \quad G_{3}\left(\frac{\partial \widetilde{\mathbf{u}}_{N}}{\partial \xi} \frac{\partial \widetilde{\mathbf{v}}_{N}}{\partial \eta}+\frac{\partial \widetilde{\mathbf{u}}_{N}}{\partial \eta} \frac{\partial \widetilde{\mathbf{v}}_{N}}{\partial \xi}\right),
$$

with one variable fixed, either belong to the polynomial space $\mathbb{P}_{2 N-1}(\Lambda)^{2}$, or are bounded by a sum of the square of a polynomial in $\mathbb{P}_{N}(\Lambda)^{2}$. As a result, we can apply the GaussLobatto quadrature (2.18) and norm equivalence (2.19) to derive the following continuity inequality:

$$
\left|a_{N}\left(\mathbf{u}_{N}, \mathbf{v}_{N}\right)\right| \lesssim\left\|\mathbf{u}_{N}\right\|_{1}\left\|\mathbf{v}_{N}\right\|_{1}, \quad \forall \mathbf{u}_{N}, \mathbf{v}_{N} \in \mathbf{X}_{N} .
$$

By taking $\mathbf{v}_{N}=\mathbf{u}_{N}$ in the above, the proof of the coercivity of $a_{N}(\cdot, \cdot)$ follows from a similar argument.

Next, we establish the continuity of the bilinear form $b_{N}$. 


\section{Lemma 3.2.}

$$
\left|b_{N}\left(\mathbf{v}_{N}, q_{N}\right)\right| \lesssim\left\|\mathbf{v}_{N}\right\|_{1}\left\|q_{N}\right\|, \quad \forall \mathbf{v}_{N} \in \mathbf{X}_{N}, \forall q_{N} \in M_{N} .
$$

Proof. Using the Duffy mapping, we have

$$
\begin{aligned}
b(\mathbf{v}, q) & =-\int_{\triangle} \partial_{x} v_{1} q d x d y-\int_{\triangle} \partial_{y} v_{2} q d x d y \\
& =-\int_{\square} \frac{4}{1-\eta} \partial_{\xi} \widetilde{v_{1}} \widetilde{q} \frac{1-\eta}{8} d \xi d \eta-\int_{\square}\left(\frac{2(1+\xi)}{1-\eta} \partial_{\xi} \widetilde{v}_{2}+2 \partial_{\eta} \widetilde{v}_{2}\right) \widetilde{q} \frac{1-\eta}{8} d \xi d \eta \\
& =-\frac{1}{2} \int_{\square} \partial_{\xi} \widetilde{v}_{1} \widetilde{q} d \xi d \eta-\int_{\square}\left(\frac{1+\xi}{4} \partial_{\xi} \widetilde{v}_{2}+\frac{1-\eta}{4} \partial_{\eta} \widetilde{v}_{2}\right) \widetilde{q} d \xi d \eta .
\end{aligned}
$$

It is readily seen that the integrands are all polynomials of degree less than $2 N-1$ for all $\mathbf{v} \in \mathbf{X}_{N}, q \in M_{N}$. Thus the Gauss-Lobatto quadrature applied to the above integrals is exact for such functions, which results in

$$
b(\mathbf{v}, q)=-\left(\partial_{x} v_{1}, q\right)_{N}-\left(\partial_{y} v_{2}, q\right)_{N}=b_{N}(\mathbf{v}, q), \quad \forall \mathbf{v} \in \mathbf{X}_{N}, \quad \forall q \in M_{N} .
$$

Consequently, we obtain

$$
\left|b_{N}\left(\mathbf{v}_{N}, q_{N}\right)\right|=\left|b\left(\mathbf{v}_{N}, q_{N}\right)\right| \leq\left\|\mathbf{v}_{N}\right\|_{1}\left\|q_{N}\right\|, \quad \forall \mathbf{v}_{N} \in \mathbf{X}_{N}, \quad \forall q_{N} \in M_{N} .
$$

The following lemma guarantees the uniqueness of the pressure.

\section{Lemma 3.3.}

$$
\left\{q_{N} \in M_{N}: b_{N}\left(\mathbf{v}_{N}, q_{N}\right)=0, \forall \mathbf{v}_{N} \in \mathbf{X}_{N}\right\}=\{0\} .
$$

Proof. First, for all $\mathbf{v}_{N} \in \mathbf{X}_{N}, q_{N} \in M_{N}$, we have

$$
\begin{aligned}
\int_{\triangle} \partial_{x} v_{1, N} q_{N} d x d y & =\int_{\square} \frac{4}{1-\eta} \partial_{\xi} \widetilde{v}_{1, N} \widetilde{q}_{N} \frac{1-\eta}{8} d \xi d \eta \\
& =\frac{1}{2} \int_{\square} \partial_{\xi} \widetilde{v}_{1, N} \widetilde{q}_{N} d \xi d \eta \\
& =-\frac{1}{2} \int_{\square} \widetilde{v}_{1, N} \partial_{\xi} \widetilde{q}_{N} d \xi d \eta \\
& =-\int_{\triangle} v_{1, N} \partial_{x} q_{N} d x d y,
\end{aligned}
$$

and

$$
\begin{aligned}
\int_{\triangle} \partial_{y} v_{2, N} q_{N} d x d y & =\int_{\square}\left(\frac{2(1+\xi)}{1-\eta} \partial_{\xi} \widetilde{v}_{2, N}+2 \partial_{\eta} \widetilde{v}_{2, N}\right) \frac{1-\eta}{8} \widetilde{q}_{N} d \xi d \eta \\
& =\int_{\square}\left(\frac{1+\xi}{4} \partial_{\xi} \widetilde{v}_{2, N}+\frac{1-\eta}{4} \partial_{\eta} \widetilde{v}_{2, N}\right) \widetilde{q}_{N} d \xi d \eta
\end{aligned}
$$




$$
\begin{aligned}
& =\int_{\square} \widetilde{v}_{2, N}\left(\frac{1}{4} \widetilde{q}_{N}+\frac{1+\xi}{4} \partial_{\xi} \widetilde{q}_{N}-\frac{1}{4} \widetilde{q}_{N}+\frac{1-\eta}{4} \partial_{\eta} \widetilde{q}_{N}\right) d \xi d \eta \\
& =-\int_{\square} \widetilde{v}_{2, N}\left(\frac{1+\xi}{4} \partial_{\xi} \widetilde{q}_{N}+\frac{1-\eta}{4} \partial_{\eta} \widetilde{q}_{N}\right) d \xi d \eta \\
& =-\int_{\triangle} v_{2, N} \partial_{y} q_{N} d x d y .
\end{aligned}
$$

It can be directly verified that

$$
\begin{aligned}
\left\{\widetilde{\mathbf{v}} ; \widetilde{\mathbf{v}}=\mathbf{v} \circ \mathbf{F}, \mathbf{v} \in \mathbf{X}_{N}\right\} & =\left\{\widetilde{\mathbf{v}} \in\left(\widetilde{\mathbb{Q}}_{N}^{0}(\square)\right)^{2}, \partial_{\xi} \widetilde{\mathbf{v}}(\xi, 1)=0\right\} \\
& =\operatorname{span}\left\{J_{i}^{0,0}(\xi) J_{j}^{1,0}(\eta), i, j=1, \cdots, N-1\right\} \\
& =\operatorname{span}\left\{h_{i}(\xi) h_{j}(\eta), i, j=1, \cdots, N-1\right\},
\end{aligned}
$$

where $h_{i}(\xi)$ is the Lagrangian interpolation polynomial based on the $N+1$ LegendreGauss-Lobatto points $\xi_{i}, i=0,1, \cdots, N$.

Let $q_{N} \in M_{N}$ be a function such that $b_{N}\left(\mathbf{v}_{N}, q_{N}\right)=0, \forall \mathbf{v}_{N} \in \mathbf{X}_{N}$. Then, by taking

$$
\widetilde{\mathbf{v}}_{N}=\left(\widetilde{v}_{1, N}, \widetilde{v}_{2, N}\right)^{T}=\left(h_{i}(\xi) h_{j}(\eta), 0\right)^{T}, \quad i, j=1, \cdots, N-1,
$$

we have, in virtue of (3.1) and (3.2),

$$
\begin{aligned}
0 & =b_{N}\left(\mathbf{v}_{N}, q_{N}\right)=b\left(\mathbf{v}_{N}, q_{N}\right) \\
& =-\int_{\triangle} \partial_{x} v_{1, N} q_{N} d x d y=\int_{\triangle} v_{1, N} \partial_{x} q_{N} d x d y \\
& =\int_{\square} \widetilde{v}_{1, N} \frac{4}{1-\eta} \partial_{\xi} \widetilde{q}_{N} \frac{1-\eta}{8} d \xi d \eta=\frac{1}{2} \int_{\square} \widetilde{v}_{1, N} \partial_{\xi} \widetilde{q}_{N} d \xi d \eta \\
& =\frac{1}{2} \sum_{m, n=0}^{N} h_{i}\left(\xi_{m}\right) h_{j}\left(\eta_{n}\right) \partial_{\xi} \widetilde{q}_{N}\left(\xi_{m}, \xi_{n}\right) \omega_{m} \omega_{n} \\
& =\frac{1}{2} \partial_{\xi} \widetilde{q}_{N}\left(\xi_{i}, \xi_{j}\right) \omega_{i} \omega_{j}, \quad \forall i, j=1, \cdots, N-1 .
\end{aligned}
$$

The above implies that $\partial_{\xi} \widetilde{q}_{N}\left(\xi_{i}, \eta\right)=0$ at $N-1$ distinct points in $\eta$. Hence $\partial_{\xi} \widetilde{q}_{N}\left(\xi_{i}, \eta\right)=0$ for all $\eta \in \Lambda$, since $\partial_{\xi} \widetilde{q}_{N}\left(\xi_{i}, \eta\right)$ is a polynomial of degree $\leq N-2$ in $\eta$. Similarly we have $\partial_{\xi} \widetilde{q}_{N}(\xi, \eta)=0$ for all $\xi \in \Lambda$, since $\partial_{\xi} \widetilde{q}_{N}(\xi, \eta)$ is a polynomial of degree $\leq N-3$ in $\xi$, and vanishes at $N-1$ distinct points in the same variable. Thus

$$
\partial_{\xi} \widetilde{q}_{N}(\xi, \eta)=0, \quad \forall(\xi, \eta) \in \Lambda^{2} .
$$

Hence, we have shown that $\widetilde{q}_{N}$ is independent of the variable $\xi$.

On the other side, by taking

$$
\widetilde{\mathbf{v}}_{N}=\left(\widetilde{v}_{1, N}, \widetilde{v}_{2, N}\right)^{T}=\left(0, h_{i}(\xi) h_{j}(\eta)\right)^{T}, \quad i, j=1, \cdots, N-1,
$$


and using (3.1), (3.3), and (3.4), we obtain

$$
\begin{aligned}
0 & =\int_{\triangle} v_{2, N} \partial_{y} q_{N} d x d y \\
& =\int_{\square} \widetilde{v}_{2, N}\left(\frac{2(1+\xi)}{1-\eta} \partial_{\xi} \widetilde{q}_{N}+2 \partial_{\eta} \widetilde{q}_{N}\right) \frac{1-\eta}{8} d \xi d \eta \\
& =\int_{\square}^{N} \widetilde{v}_{2, N} \frac{1-\eta}{4} \partial_{\eta} \widetilde{q}_{N} d \xi d \eta \\
& =\sum_{m, n=0}^{N} h_{i}\left(\xi_{m}\right) h_{j}\left(\eta_{n}\right) \partial_{\eta} \widetilde{q}_{N}\left(\xi_{m}, \eta_{n}\right) \frac{1-\eta_{n}}{4} \omega_{m} \omega_{n} \\
& =\partial_{\eta} \widetilde{q}_{N}\left(\xi_{i}, \eta_{j}\right) \frac{1-\eta_{j}}{4} \omega_{i} \omega_{j} .
\end{aligned}
$$

This means that for fixed $j, \partial_{\eta} \widetilde{q}_{N}\left(\xi, \eta_{j}\right)=0$ at $N-1$ distinct points in $\xi$, and therefore $\partial_{\eta} \widetilde{q}_{N}\left(\xi, \eta_{j}\right)=0, \forall \xi \in \Lambda$. For the same reason, we have

$$
\partial_{\eta} \widetilde{q}_{N}(\xi, \eta)=0, \quad \forall(\xi, \eta) \in \Lambda^{2}
$$

Combining this result and (3.4), and noting that $\int_{\triangle} q_{N} d x d y=0$, we conclude

$$
q_{N}(x, y)=\widetilde{q}_{N}(\xi, \eta) \equiv 0 .
$$

The lemma is proved.

Since $\mathbf{X}_{N}$ and $M_{N}$ are finite dimensional, an immediate consequence of the above lemma is that there exists $\beta_{N}>0$ such that

$$
\inf _{q_{N} \in M_{N}} \sup _{\mathbf{v}_{N} \in \mathbf{X}_{N}} \frac{b_{N}\left(\mathbf{v}_{N}, q_{N}\right)}{\left\|\mathbf{v}_{N}\right\|_{1}\left\|q_{N}\right\|} \geq \beta_{N}
$$

However, an explicit lower bound for $\beta_{N}$ appears to be illusive. Nevertheless, the above results and Lemmas 3.1 and 3.2 ensure that the discrete problem (2.22) admits a unique solution.

\subsection{Error estimation for the velocity}

Let $Y_{N}=H_{0}^{2}(\triangle) \cap \mathbb{Q}_{N}(\triangle)$. First we remark that the space $Y_{N}$ can be characterized by

$$
\begin{aligned}
\widetilde{Y}_{N} & :=\left\{\widetilde{v}: \widetilde{v}=v \circ \mathbf{F}, v \in Y_{N}\right\} \\
& =\left\{\widetilde{v}: \widetilde{v} \in \widetilde{\mathbb{Q}}_{N}(\square),\left.\widetilde{v}\right|_{\partial \square}=0,\left.\frac{\partial \widetilde{v}}{\partial n}\right|_{\partial \square}=0, \partial_{\xi} \widetilde{v}(\xi, 1)=0, \partial_{\xi}^{2} \widetilde{v}(\xi, 1)=0\right\} \\
& =\operatorname{span}\left\{(1-\xi)^{2}(1+\xi)^{2}(1-\eta)^{2}(1+\eta)^{2} J_{l}^{2,2}(\xi) J_{m}^{2,2}(\eta), 0 \leq l, m \leq N-4\right\} .
\end{aligned}
$$


Then we define the $H_{0}^{2}$-orthogonal projector $\pi_{N}^{2,0}: H_{0}^{2}(\triangle) \rightarrow Y_{N}$ by: $\forall v \in H_{0}^{2}(\triangle)$,

$$
\left(\Delta\left(\pi_{N}^{2,0} v-v\right), \Delta \varphi\right)=0, \quad \forall \varphi \in Y_{N}
$$

An error estimate for the projector $\pi_{N}^{2,0}$ is derived in the following lemma.

Lemma 3.4. For all $v \in H_{0}^{2}(\triangle) \cap H^{s}(\triangle)$ with $s \geq 2$, it holds

$$
\left\|\pi_{N}^{2,0} v-v\right\|_{2} \lesssim N^{3-s}\|v\|_{s} .
$$

Proof. By the definition of $\pi_{N}^{2,0}$, immediately we have, $\forall v \in H_{0}^{2}(\triangle) \cap H^{s}(\triangle)$,

$$
\left\|\pi_{N}^{2,0} v-v\right\|_{2} \lesssim\left\|\Delta\left(\pi_{N}^{2,0} v-v\right)\right\| \leq \inf _{\varphi \in Y_{N}}\|\Delta(\varphi-v)\| .
$$

In order to derive an error estimate for the right hand side, we will use Theorem 4.2 in [10]. More precisely, let

$$
Y_{N}^{*}=\operatorname{span}\left\{x^{k} y^{l}: k, l \in \mathbb{N}, k+l \leq N\right\} \cap H_{0}^{2}(\triangle),
$$

we obtain from Theorem 4.2 in [10] that

$$
\inf _{\varphi \in Y_{N}^{*}}|\varphi-v|_{2} \lesssim N^{3-s}\|v\|_{s}
$$

On the other hand, it can be verified that the transformation in $\square$ of $Y_{N}^{*}$ is

$$
\widetilde{Y}_{N}^{*}=\operatorname{span}\left\{(1-\xi)^{2}(1+\xi)^{2}(1-\eta)^{l}(1+\eta)^{2} J_{l-4}^{2,2}(\xi) J_{m-2}^{2 l-3,2}(\eta), l \geq 4, m \geq 2, l+m \leq N\right\} .
$$

A direct comparison with (3.5) yields the following inclusion:

$$
\widetilde{Y}_{N}^{*} \subset \widetilde{Y}_{N}
$$

which implies that

$$
Y_{N}^{*} \subset Y_{N}
$$

Finally, we obtain

$$
\left\|\pi_{N}^{2,0} v-v\right\|_{2} \lesssim \inf _{\varphi \in Y_{N}}\|\Delta(\varphi-v)\| \leq \inf _{\varphi \in Y_{N}^{*}}\|\Delta(\varphi-v)\| \lesssim \inf _{\varphi \in Y_{N}^{*}}|\varphi-v|_{2} \lesssim N^{3-s}\|v\|_{s} .
$$

This completes the proof of the lemma.

Remark 3.1. We note that the error estimate in Lemma 3.4 is suboptimal due to the use of Theorem 4.2 in [10]. As a consequence, all subsequent results in this section are also suboptimal. It is likely that this result can be improved by using a more delicate analysis (see Remark 4.1 in [10]). 
Next, we derive an approximation result for divergence-free spaces. Let $\mathbf{V}$ and $\mathbf{V}_{N}$ be respectively

$$
\begin{aligned}
& \mathbf{V}=\{\mathbf{v} \in \mathbf{X}: \forall q \in M, b(\mathbf{v}, q)=0\} \\
& \mathbf{V}_{N}=\left\{\mathbf{v}_{N} \in \mathbf{X}_{N}: \forall q_{N} \in M_{N}, b_{N}\left(\mathbf{v}_{N}, q_{N}\right)=0\right\}
\end{aligned}
$$

Lemma 3.5. For any $\mathbf{v} \in H^{s}(\triangle)^{2} \cap \mathbf{V}, s \geq 2$, it holds

$$
\inf _{\mathbf{v}_{N} \in \mathbf{V}_{N}}\left\|\mathbf{v}-\mathbf{v}_{N}\right\|_{1} \lesssim N^{2-s}\|\mathbf{v}\|_{s}
$$

Proof. First of all, we recall that (cf. [1]), for any $\mathbf{v} \in H^{s}(\triangle)^{2} \cap \mathbf{V}, s \geq 2$, there exists a stream function $\psi \in H^{s+1}(\triangle)$ such that

$$
\mathbf{v}=\nabla \times \psi:=\left(\frac{\partial \psi}{\partial y},-\frac{\partial \psi}{\partial x}\right), \text { in } \triangle
$$

and

$$
\|\psi\|_{s+1} \lesssim\|\mathbf{v}\|_{s} .
$$

Moreover we have $\nabla \psi=0$ on $\partial \Delta$ due to the fact that $\mathbf{v}=0$ on $\partial \triangle$. As a result, we have $\left.(\partial \psi / \partial n)\right|_{\partial \triangle}=0$, and $\left.\psi\right|_{\partial \Delta}=c$, where $c$ is a constant. Since $\psi$ is determined up to an additive constant, we can take a $\psi$ such that $\left.\psi\right|_{\partial \Delta}=0$. Thus $\psi \in H_{0}^{2}(\triangle)$. Now let $\pi_{N}^{2,0}$ be the projector defined in (3.6), then by Lemma 3.4, we have

$$
\left\|\psi-\pi_{N}^{2,0} \psi\right\|_{2} \lesssim N^{2-s}\|\psi\|_{s+1} \lesssim N^{2-s}\|\mathbf{v}\|_{s}, \quad s \geq 2 .
$$

Furthermore, by definition of $\pi_{N}^{2,0}, \overline{\pi_{N}^{2,0} \psi}$ belongs to the space $\widetilde{Y}_{N}$, defined in (3.5). Now we take $\mathbf{v}_{N}=\nabla \times\left(\pi_{N}^{2,0} \psi\right)$, then it is readily seen that $\widetilde{\mathbf{v}}_{N} \in \widetilde{\mathbb{Q}}_{N}^{0}(\square)$, and $\partial_{\xi} \widetilde{\mathbf{v}}_{N}(\xi, 1)=0$. Therefore, we have $\mathbf{v}_{N} \in \mathbf{X}_{N}$, and so that $\mathbf{v}_{N} \in \mathbf{V}_{N}$. By using (3.8), we conclude that for any $\mathbf{v} \in H^{s}(\triangle)^{2} \cap \mathbf{V}, s \geq 2$, we have

$$
\inf _{\mathbf{v}_{N} \in \mathbf{V}_{N}}\left\|\mathbf{v}-\mathbf{v}_{N}\right\|_{1} \leq\left\|\nabla \times \psi-\nabla \times\left(\pi_{N}^{2,0} \psi\right)\right\|_{1} \leq\left\|\psi-\pi_{N}^{2,0} \psi\right\|_{2} \lesssim N^{2-s}\|\mathbf{v}\|_{s} .
$$

This completes the proof of the lemma.

We are now in position to prove the main result.

Theorem 3.1. Let $\mathbf{u}$ be the velocity solution of problem (2.16), $\mathbf{u}_{N}$ be the discrete solution of the triangular spectral approximation (2.22). If $\mathbf{u} \in H^{s}(\triangle)^{2}, s \geq 2$, then it holds

$$
\left\|\mathbf{u}-\mathbf{u}_{N}\right\|_{1} \lesssim N^{2-s}\|\mathbf{u}\|_{s} .
$$

Proof. Let $\mathbf{w}_{N}$ be an arbitrary function in $\mathbf{V}_{N}$. Setting $\boldsymbol{\sigma}_{N}:=\mathbf{u}_{N}-\mathbf{w}_{N}$, and using Lemma 3.1, we have

$$
\alpha\left\|\boldsymbol{\sigma}_{N}\right\|_{1}^{2} \leq a_{N}\left(\boldsymbol{\sigma}_{N}, \boldsymbol{\sigma}_{N}\right)=a\left(\mathbf{u}-\mathbf{w}_{N}, \boldsymbol{\sigma}_{N}\right)+a\left(\mathbf{w}_{N}, \boldsymbol{\sigma}_{N}\right)-a_{N}\left(\mathbf{w}_{N}, \boldsymbol{\sigma}_{N}\right) .
$$


Furthermore, using the continuity of $a(\cdot, \cdot)$ yields

$$
\alpha\left\|\boldsymbol{\sigma}_{N}\right\|_{1} \leq \gamma\left\|\mathbf{u}-\mathbf{w}_{N}\right\|_{1}+\sup _{\substack{\mathbf{v}_{N} \in \mathbf{X}_{N} \\ \mathbf{v}_{N} \neq 0}} \frac{\left|a\left(\mathbf{w}_{N}, \mathbf{v}_{N}\right)-a_{N}\left(\mathbf{w}_{N}, \mathbf{v}_{N}\right)\right|}{\left\|\mathbf{v}_{N}\right\|_{1}} .
$$

Combining the above inequality with the triangular inequality

$$
\left\|\mathbf{u}-\mathbf{u}_{N}\right\|_{1} \leq\left\|\mathbf{u}-\mathbf{w}_{N}\right\|_{1}+\left\|\boldsymbol{\sigma}_{N}\right\|_{1},
$$

we find

$$
\left\|\mathbf{u}-\mathbf{u}_{N}\right\|_{1} \leq\left(1+\frac{\gamma}{\alpha}\right)\left\|\mathbf{u}-\mathbf{w}_{N}\right\|_{1}+\frac{1}{\alpha} \sup _{\substack{\mathbf{v}_{N} \in \mathbf{X}_{N} \\ \mathbf{v}_{N} \neq 0}} \frac{\left|a\left(\mathbf{w}_{N}, \mathbf{v}_{N}\right)-a_{N}\left(\mathbf{w}_{N}, \mathbf{v}_{N}\right)\right|}{\left\|\mathbf{v}_{N}\right\|_{1}}, \quad \forall \mathbf{w}_{N} \in \mathbf{V}_{N} .
$$

Thanks to (2.18), we readily verify that

$$
a_{N}\left(\mathbf{w}_{N}, \mathbf{v}_{N}\right)=a\left(\mathbf{w}_{N}, \mathbf{v}_{N}\right), \quad \forall \mathbf{w}_{N} \in \mathbf{X}_{N-1}, \quad \forall \mathbf{v}_{N} \in \mathbf{X}_{N} .
$$

Thus we have

$$
\begin{aligned}
& \left\|\mathbf{u}-\mathbf{u}_{N}\right\|_{1} \\
\leq & \inf _{\mathbf{w}_{N} \in \mathbf{V}_{N}}\left(\left(1+\frac{\gamma}{\alpha}\right)\left\|\mathbf{u}-\mathbf{w}_{N}\right\|_{1}+\frac{1}{\alpha} \sup _{\substack{\mathbf{v}_{N} \in \mathbf{X}_{N} \\
\mathbf{v}_{N} \neq 0}} \frac{\left|a\left(\mathbf{w}_{N}, \mathbf{v}_{N}\right)-a_{N}\left(\mathbf{w}_{N}, \mathbf{v}_{N}\right)\right|}{\left\|\mathbf{v}_{N}\right\|_{1}}\right) \\
\leq & \inf _{\mathbf{w}_{N} \in \mathbf{V}_{N} \cap \mathbf{x}_{N-1}}\left(\left(1+\frac{\gamma}{\alpha}\right)\left\|\mathbf{u}-\mathbf{w}_{N}\right\|_{1}\right) .
\end{aligned}
$$

Finally, by virtue of Lemma 3.5, we conclude that

$$
\left\|\mathbf{u}-\mathbf{u}_{N}\right\|_{1} \lesssim N^{2-s}\|\mathbf{u}\|_{s} .
$$

This completes the proof of the theorem.

\section{Implementation with a nodal basis}

In this section we describe the implementation details of our triangular spectral method, based on a simple nodal basis for the velocity and pressure approximation spaces.

Under the Duffy mapping, the approximation spaces $X_{N}, M_{N}$ in $\triangle$ are transformed into:

$$
\begin{aligned}
\widetilde{X}_{N} & :=\widetilde{\mathbb{Q}}_{N}^{0}(\square) \cap \widetilde{H}_{\varpi}^{1}(\square)=\left\{\varphi \in \widetilde{\mathbb{Q}}_{N}^{0}(\square), \partial_{\xi} \varphi(\xi, 1)=0\right\}, \\
\widetilde{M}_{N} & :=\widetilde{\mathbb{Q}}_{N-2}(\square) \cap L_{0, \varpi}^{2}(\square),
\end{aligned}
$$

where $\varpi$ is defined in (2.12), $\widetilde{\mathbb{Q}}_{N}(\square)$ and $\widetilde{H}_{\varpi}^{1}(\square)$ are respectively given in (2.10) and (2.13). 
We now observe the following basic facts:

$$
\begin{aligned}
& \widetilde{\mathbb{Q}}_{N}(\square)=\operatorname{span}\left\{h_{i}(\xi) h_{j}(\eta), 0 \leq i, j \leq N\right\}, \\
& \widetilde{\mathbb{Q}}_{N}^{0}(\square)=\operatorname{span}\left\{h_{i}(\xi) h_{j}(\eta), 1 \leq i, j \leq N-1\right\}, \\
& \widetilde{X}_{N}=\widetilde{\mathbb{Q}}_{N}^{0}(\square), \\
& \widetilde{M}_{N}=\operatorname{span}\left\{l_{i}(\xi) l_{j}(\eta), 1 \leq i, j \leq N-1\right\} \cap L_{0, \varpi}^{2}(\square),
\end{aligned}
$$

where $l_{p}(\xi), p=1, \cdots, N-1$, are the one-dimensional Lagrangian interpolants through the interior Legendre-Gauss-Lobatto points, such that $l_{p}(\xi) \in \mathbb{P}_{N-2}(\Lambda), l_{p}\left(\xi_{q}\right)=\delta_{p q}, 1 \leq$ $p, q \leq N-1$.

Let $\widetilde{\varphi}_{i j}(\xi, \eta)=h_{i}(\xi) h_{j}(\eta), \widetilde{\psi}_{i j}(\xi, \eta)=l_{i}(\xi) l_{j}(\eta)$, and define the basis functions

$$
\varphi_{i j}(x, y)=\tilde{\varphi}_{i j}(\xi, \eta), \psi_{i j}(x, y)=\widetilde{\psi}_{i j}(\xi, \eta)
$$

through the Duffy mapping, we have

$$
\begin{aligned}
& \mathbf{X}_{N}=\operatorname{span}\left\{\left(\varphi_{i j}(x, y), 0\right),\left(0, \varphi_{i j}(x, y)\right): 1 \leq i, j \leq N-1\right\}, \\
& M_{N}=\operatorname{span}\left\{\psi_{i j}(x, y), 1 \leq i, j \leq N-1\right\} \cap L_{0}^{2}(\triangle) .
\end{aligned}
$$

Therefore, we can write

$$
\begin{aligned}
& \mathbf{u}_{N}(x, y)=\widetilde{\mathbf{u}}_{N}(\xi, \eta)=\sum_{i, j=1}^{N-1} \mathbf{u}_{i j} \widetilde{\varphi}_{i j}(\xi, \eta), \\
& p_{N}(x, y)=\widetilde{p}_{N}(\xi, \eta)=\sum_{i, j=1}^{N-1} p_{i j} \widetilde{\psi}_{i j}(\xi, \eta),
\end{aligned}
$$

where, by definition of the basis functions, $\mathbf{u}_{i j}=\widetilde{\mathbf{u}}_{N}\left(\xi_{i}, \xi_{j}\right)=\mathbf{u}_{N}\left(x_{i}, y_{j}\right), p_{i j}=\widetilde{p}_{N}\left(\xi_{i}, \xi_{j}\right)=$ $p_{N}\left(x_{i}, y_{j}\right), 1 \leq i, j \leq N-1$, with $\left(x_{i}, y_{j}\right)$ the mapped points in $\Delta$ of the Gauss-Lobatto points $\left(\xi_{i}, \xi_{j}\right)$, i.e., $\left(x_{i}, y_{j}\right)=\mathbf{F}\left(\xi_{i}, \xi_{j}\right)$; see Fig. 2 .
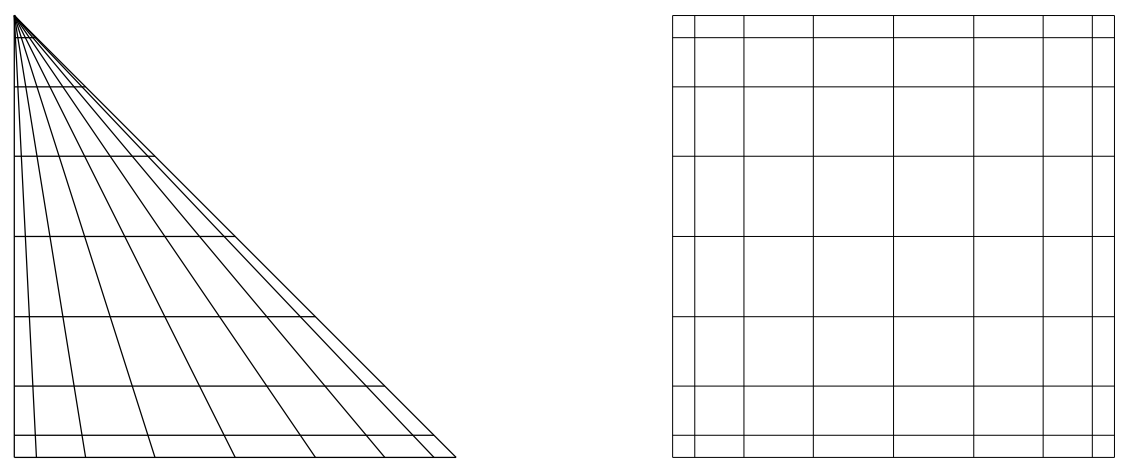

Figure 2: Mapping of the triangle to square domain. 
Plugging these expressions into (2.22), and letting $\mathbf{v}_{N}$ and $q_{N}$ to run through all the basis functions $\left(\varphi_{i j}, 0\right),\left(0, \varphi_{i j}\right)$ and $\psi_{i j}$ respectively, we arrive at the following matrix system:

$$
\left\{\begin{array}{l}
\mathbf{A}_{N} \underline{\mathbf{u}}+\mathbf{D}_{N} \underline{p}=\mathbf{B}_{N} \underline{\mathbf{f}} \\
\mathbf{D}_{N}^{T} \underline{\mathbf{u}}=0,
\end{array}\right.
$$

where $\underline{\mathbf{f}}$ is a vector representation of $\mathbf{f}$ at the Gauss-Lobatto points. The matrices $\mathbf{A}_{N}, \mathbf{D}_{N}$ and $\mathbf{B}_{N}$ are block-diagonal matrices with 2 blocks each. The blocks of $\mathbf{A}_{N}$ are the discrete Laplace operator, and those of $\mathbf{D}_{N}$ are associated to the different components of the discrete gradient operators, while blocks of $\mathbf{B}_{N}$ are the diagonal mass matrices.

An important property of the nodal spectral-element method is that the matrix-vector product can be efficiently carried out thanks to the tensor product structure. In the following we give a detailed description on how to perform $\mathbf{A}_{N} \mathbf{v}_{N}$ by using the tensor product factorization technique.

$$
\begin{aligned}
\sum_{i, j=1}^{N-1} a_{m n, i j} u_{i j}=\sum_{p, q=0}^{N} & \left(\frac{\left(1+\xi_{p}\right)^{2}+4}{2\left(1-\xi_{q}\right)}\left[\sum_{i, j=1}^{N-1} u_{i j} h_{i}^{\prime}\left(\xi_{p}\right) h_{j}\left(\xi_{q}\right)\right] h_{m}^{\prime}\left(\xi_{p}\right) h_{n}\left(\xi_{q}\right) \omega_{p q}\right) \\
& +\sum_{p, q=0}^{N}\left(\frac{1-\xi_{q}}{2}\left[\sum_{i, j=1}^{N-1} u_{i j} h_{i}\left(\xi_{p}\right) h_{j}^{\prime}\left(\xi_{q}\right)\right] h_{m}\left(\xi_{p}\right) h_{n}^{\prime}\left(\xi_{q}\right) \omega_{p q}\right) \\
& +\sum_{p, q=0}^{N}\left(\frac{1+\xi_{p}}{2}\left[\sum_{i, j=1}^{N-1} u_{i j} h_{i}^{\prime}\left(\xi_{p}\right) h_{j}\left(\xi_{q}\right)\right] h_{m}\left(\xi_{p}\right) h_{n}^{\prime}\left(\xi_{q}\right) \omega_{p q}\right) \\
& +\sum_{p, q=0}^{N}\left(\frac{1+\xi_{p}}{2}\left[\sum_{i, j=1}^{N-1} u_{i j} h_{i}\left(\xi_{p}\right) h_{j}^{\prime}\left(\xi_{q}\right)\right] h_{m}^{\prime}\left(\xi_{p}\right) h_{n}\left(\xi_{q}\right) \omega_{p q}\right)
\end{aligned}
$$

where $\omega_{p q}=\omega_{p} \omega_{q}$. Let's emphasize that the denominator $1-\xi_{q}$ in the first term does not cause any difficulty because the quantity behind this factor vanishes for $q=N$. In fact, a rearrangement of above expression leads to

$$
\begin{aligned}
\sum_{i, j=1}^{N-1} a_{m n, i j} u_{i j}=\sum_{p, q=0}^{N} & \left(\frac{\left(1+\xi_{p}\right)^{2}+4}{2\left(1-\xi_{q}\right)}\left[\sum_{i, j=1}^{N-1} u_{i j} D_{p i} \delta_{q j}\right] D_{p m} \delta_{q n} \omega_{p q}\right) \\
& +\sum_{p, q=0}^{N}\left(\frac{1-\xi_{q}}{2}\left[\sum_{i, j=1}^{N-1} u_{i j} \delta_{p i} D_{q j}\right] \delta_{p m} D_{q n} \omega_{p q}\right) \\
& +\sum_{p, q=0}^{N}\left(\frac{1+\xi_{p}}{2}\left[\sum_{i, j=1}^{N-1} u_{i j} D_{p i} \delta_{q j}\right] \delta_{p m} D_{q n} \omega_{p q}\right) \\
& +\sum_{p, q=0}^{N}\left(\frac{1+\xi_{p}}{2}\left[\sum_{i, j=1}^{N-1} u_{i j} \delta_{p i} D_{q j}\right] D_{p m} \delta_{q n} \omega_{p q}\right)
\end{aligned}
$$




$$
\begin{aligned}
=\sum_{p=0}^{N} & \frac{\left(1+\xi_{p}\right)^{2}+4}{2\left(1-\xi_{n}\right)} D_{p m}\left[\sum_{i=1}^{N-1} D_{p i} u_{i n}\right] \omega_{p n} \\
& +\sum_{q=0}^{N} \frac{1-\xi_{q}}{2} D_{q n}\left[\sum_{j=1}^{N-1} D_{q j} u_{m j}\right] \omega_{m q}+\sum_{q=1}^{N-1} \frac{1+\xi_{m}}{2} D_{q n}\left[\sum_{i=1}^{N-1} D_{m i} u_{i q}\right] \omega_{m q} \\
& +\sum_{p=1}^{N-1} \frac{1+\xi_{p}}{2} D_{p m}\left[\sum_{j=1}^{N-1} D_{n j} u_{p j}\right] \omega_{p n}, \quad \forall m, n=1, \cdots, N-1,
\end{aligned}
$$

where $D_{p i}=h_{i}^{\prime}\left(\xi_{p}\right)$.

\section{Numerical results and discussions}

This section is devoted to verify the accuracy of the triangular spectral method, and to investigate the numerical behavior of the inf-sup constant $\beta_{N}$ in (3.5).

\subsection{Numerical investigation of the inf-sup constant}

Since the matrix $\mathbf{A}_{N}$ is positive definite, the system (4.1) can be decoupled by a standard block Gaussian elimination, leading to the following two decoupled systems:

$$
\begin{aligned}
& \left(\mathbf{D}_{N}^{T} \mathbf{A}_{N}^{-1} \mathbf{D}_{N}\right) \underline{p}=\mathbf{D}_{N}^{T} \mathbf{A}_{N}^{-1} \mathbf{B}_{N} \underline{\mathbf{f}}, \\
& \mathbf{A}_{N} \underline{\mathbf{u}}=-\mathbf{D}_{N} \underline{p}+\mathbf{B}_{N} \underline{\mathbf{f}},
\end{aligned}
$$

where $\mathbf{S}_{N}:=\mathbf{D}_{N}^{T} \mathbf{A}_{N}^{-1} \mathbf{D}_{N}$ is the so called Schur-complement. The above systems are usually solved by using a preconditioned conjugate gradient method.

It is well-known (cf. [11]) that the condition number of the preconditioned Schurcomplement $\mathbf{B}_{N}^{-1} \mathbf{S}_{N}$ is of order $1 / \beta_{N}^{2}$. Hence, the efficiency of our method is directly linked to the behavior of $\beta_{N}$. Since we are unable to derive an estimate for the inf-sup constant $\beta_{N}$, it is therefore interesting to compute the inf-sup constant numerically and investigate its behavior with respect to the degree of the approximation spaces.

We recall that in the rectangular $\mathbb{P}_{N} \times \mathbb{P}_{N-2}$ spectral method for the 2-D Stokes equations, the inf-sup constant behaves like $\mathscr{O}\left(N^{-1 / 2}\right.$ ) (cf. [1]). In Fig. 3, we plot the variations of $\beta_{N}$ (i.e., $\sqrt{\lambda_{\min } / \lambda_{\max }}$, where $\lambda_{\min }$ and $\lambda_{\max }$ are respectively the minimum and maximum eigenvalues of $\mathbf{B}_{N}^{-1} \mathbf{S}_{N}$ ) in $\log$-log scale with respect to $N$. For comparison, the results from the rectangle $\mathbb{P}_{N} \times \mathbb{P}_{N-2}$ spectral method are also presented. While we observe that the inf-sup constants for the triangular spectral method are smaller than the ones for the rectangular spectral method for all $N$ that we tested, it appears that the decay rate of the triangular spectral method (close to $\mathscr{O}\left(N^{-1 / 4}\right)$ for the range of $N$ tested) is slower than of the rectangular spectral method. 


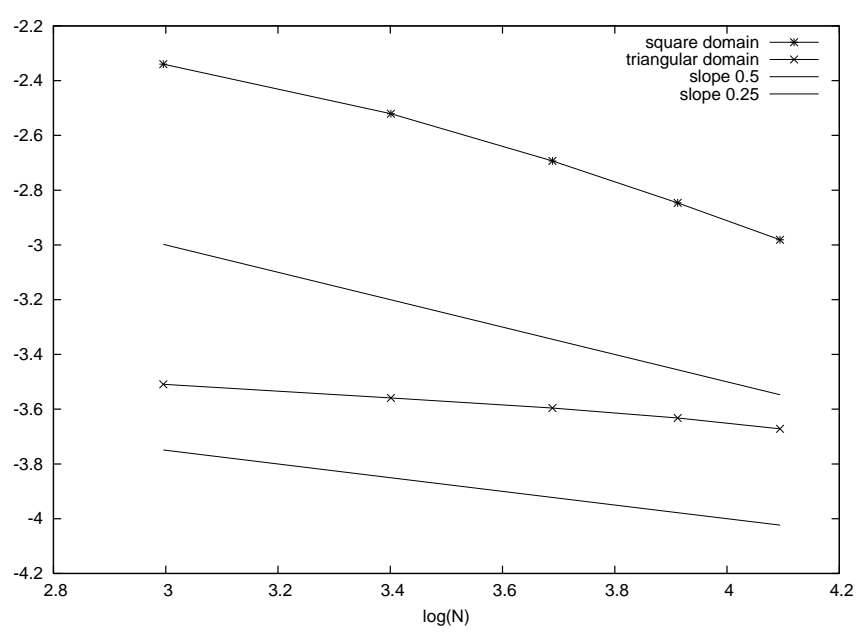

Figure 3: Plot of $\beta_{N}$ with respect to $N$ for both triangular and rectangular spectral methods.

\subsection{Accuracy tests}

It is clear that with the triangular nodal basis, one can easily include triangular elements in a spectral-element code. We now investigate the accuracy of the triangular spectral-element method with $K$ domain elements. The errors presented below are respectively in the discrete $L^{2}$-norm:

$$
\sum_{k=1}^{K}\left(\sum_{m, n=1}^{N} e_{N}^{2}\left(\xi_{m}^{k}, \xi_{n}^{k}\right) \frac{1-\xi_{n}^{k}}{8} \omega_{m n}\right)^{\frac{1}{2}}
$$

and in the discrete $H^{1}$-norm associated to the inner product $(\cdot, \cdot)_{N}+(\nabla \cdot, \nabla \cdot)_{N}$, where $e_{N}:=u_{N}-u$.

We test the method for the following exact solution:

$$
\left\{\begin{array}{l}
u_{1}(x, y)=\sin x \cos y \\
u_{2}(x, y)=-\cos x \sin y \\
p(x, y)=\sin x \sin y
\end{array}\right.
$$

in the domain $\square$, which is decomposed into four triangular and rectangular sub-domains respectively as shown in Fig. 4.

In Fig. 5, we plot, in a semi-log scale, the $L^{2}$-velocity errors (figure on the left) and the $H^{1}$-velocity errors (figure on the right). The $L^{2}$-pressure errors are presented in Fig. 6. In all these figures, the results obtained from the rectangular spectral-element method are also plotted for comparison. We observe that, as the rectangular spectral-element method, the errors for the triangular spectral-element method converge exponentially fast. We also observe that the triangular method is slightly less accurate than the rectangular method, due possibly to the unnecessarily clustered collocation points near the center. 

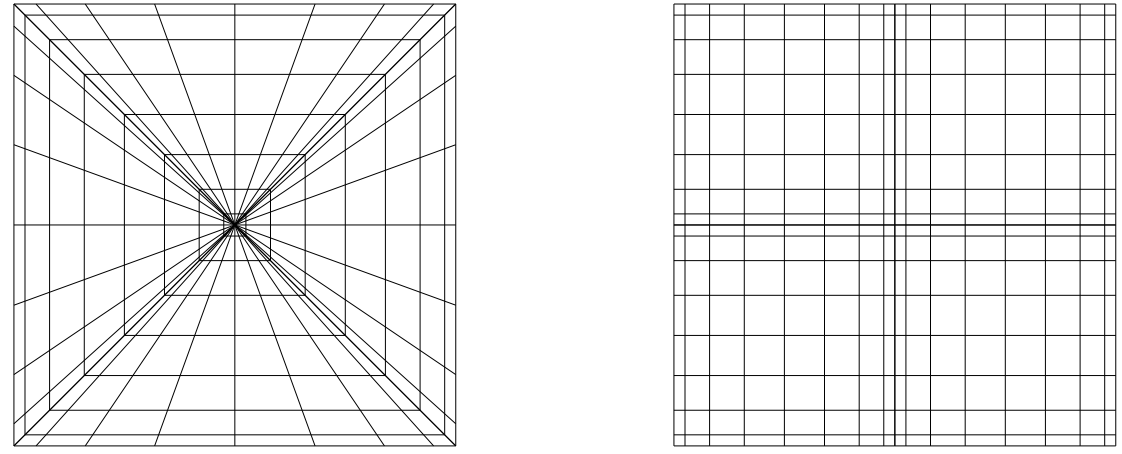

Figure 4: Triangular element mesh (left) and rectangular element mesh (right).
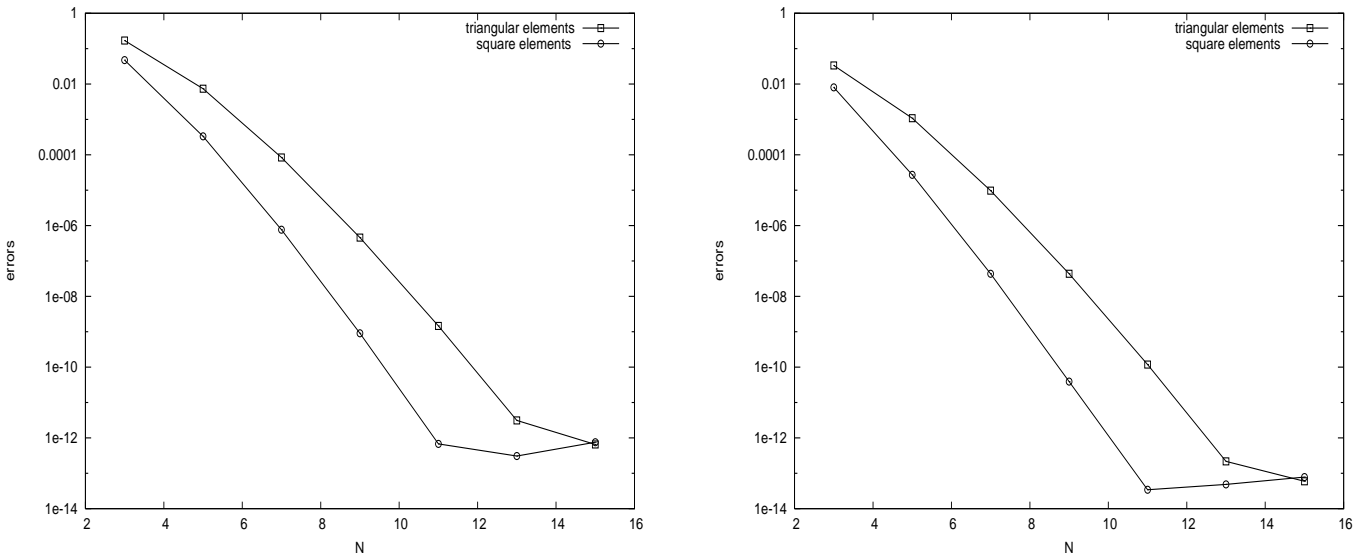

Figure 5: The velocity errors in $L^{2}$-norm (left) and $H^{1}$-norm (right) as a function of $N$.

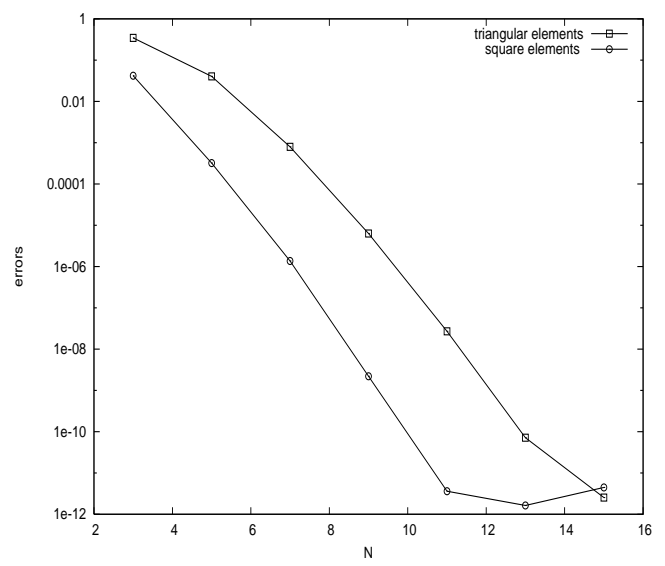

Figure 6: The pressure errors as a function of $N$. 


\subsection{Summary}

We presented a triangular spectral method based on a rational approximation through Duffy mapping for the Stokes problem. We established the well-posedness of the method and an error estimate for the velocity. Although we did not provide an explicit lower bound for the inf-sup constant, our numerical results indicates that the inf-sup constant decays at a rate between $\mathscr{O}\left(N^{-1 / 2}\right)$ and $\mathscr{O}\left(N^{-1 / 4}\right)$ for the range of $N$ we tested, and the convergence was exponential for smooth solutions and comparable to that for the rectangular spectral method. We also constructed an easy-to-implement nodal basis which leads to an efficient implementation of our method for the Stokes problem.

Acknowledgments The work of J.S. was partially supported by NFS grant DMS-0915066. The work of C.X. was partially supported by the National Natural Scheme Foundation of China (Grant number 11071203).

\section{References}

[1] C. Bernardi and Y. Maday. Approximations Spectrales de Problèmes aux Limites Elliptiques. Springer-Verlag, Paris, 1992.

[2] J. P. Boyd. Chebyshev and Fourier Spectral Methods. Springer-Verlag, 1989.

[3] D. Braess and C. Schwab. Approximation on simplices with respect to weighted sobolev norms. J. Approx. Theory, 103(2):329íc337, 2000.

[4] C. Canuto, M. Y. Hussaini, A. Quarteroni, and T. A. Zang. Spectral methods. Scientific Computation. Springer-Verlag, Berlin, 2006. Fundamentals in single domains.

[5] M. Dubiner. Spectral methods on triangles and other domains. Journal of Scientific Computing, 6(4):345-390, 1991.

[6] W. Heinrichs and B. I. Loch. Spectral schemes on triangular elements. J. Comput. Phys., 173(1):279íc301, 2001.

[7] J. S. Hesthaven. From electrostatics to almost optimal nodal sets for polynomial interpolation in a simplex. SIAM Journal of Numerical Analysis, pages 655-676, 1998.

[8] G. E. Karniadakis and S. J. Sherwin. Spectral/hp element methods for CFD. Oxford University Press, 1999.

[9] Tom Koornwinder. Two-variable analogues of the classical orthogonal polynomials. In Theory and application of special functions (Proc. Advanced Sem., Math. Res. Center, Univ. Wisconsin, Madison, Wis., 1975), pages 435-495. Math. Res. Center, Univ. Wisconsin, Publ. No. 35. Academic Press, New York, 1975.

[10] H. Li and J. Shen. Optimal error estimates in Jacobi-weighted Sobolev spaces for polynomial approximations on the triangle. Math. Comp., 79:1621-1646, 2010.

[11] Y. Maday, D. Meiron, A. T. Patera, and E. M. Rønquist. Analysis of iterative methods for the steady and unsteady Stokes problem: Application to spectral element discretizations. SIAM Journal on Scientific Computing, 14:310, 1993.

[12] R. G. Owens. Spectral approximations on the triangle. Proceedings: Mathematical, Physical and Engineering Sciences, pages 857-872, 1998.

[13] R. Pasquetti and F. Rapetti. Spectral element methods on triangles and quadrilaterals: comparisons and applications. Journal of Computational Physics, 198(1):349-362, 2004.

[14] R. Pasquetti and F. Rapetti. Spectral element methods on unstructured meshes: Comparisons and recent advances. J. Sci. Comput., 27(1-3):377-387, 2006. 
[15] Jie Shen, Li-Lian Wang, and Huiyuan Li. A triangular spectral element method using fully tensorial rational basis functions. SIAM J. Numer. Anal., 47(3):1619-1650, 2009.

[16] S. J. Sherwin and G. E. Karniadakis. A triangular spectral element method: applications to the incompressible Navier-Stokes equations. Computer Methods in Applied Mechanics and Engineering, 123(1-4):189-229, 1995.

[17] M. A. Taylor, B. A. Wingate, and R.E. Vincent. An algorithm for computing Fekete points in the triangle. SIAM Journal on Numerical Analysis, pages 1707-1720, 2001. 